\title{
A Multi-modulus Blind Equalization Algorithm Based on Memetic Algorithm
}

\author{
Guo Yecai ${ }^{1,2, \text { a }}$, Wu Xing $^{1}$, Zhang Miaoqing ${ }^{1}$ \\ ${ }^{1}$ College of Electronic and Information Engineering, Nanjing University of Information Science and \\ Technology, Nanjing 210044, China \\ ${ }^{2}$ Jiangsu Collaborative Innovation Center on Atmospheric Environment and Equipment Technology \\ (CICAEET), Nanjing 210044, China \\ aemail: guo-yecai@163.com
}

\begin{abstract}
Keywords: Signal Processing; Multi-Modulus Algorithm; Memetic Algorithm; Intelligence Optimization Algorithm; Optimal Weight Vector
\end{abstract}

\begin{abstract}
In view of slow convergence speed, large steady mean square error (MSE), and existing blind phase for the constant modulus blind equalization algorithm(CMA), a multi-modulus blind equalization algorithm based on memetic algorithm(MA-MMA) is proposed, which combines the basic idea of intelligent optimization algorithm and introduces the individual own evolution and social behavior among individuals to the blind equalization technology. In this proposed algorithm, the reciprocal of the cost function of multi-modulus blind equalization algorithm(MMA) is defined as the fitness function of the memetic algorithm(MA), the initial optimal weight vector of the MMA is optimized by using the global information sharing mechanism and local depth search ability of the MA. When the initial optimum weight vector of the MMA is obtained, the weight vector of the MMA may be updated. The simulation results with the higher-order APSK multi-modulus signals show that, compared with the CMA, the MMA, and the multi-modulus blind equalization algorithm based on genetic algorithm(GA-MMA), the proposed MA-MMA has the fastest convergence speed, smallest mean square error(MSE), and clearest output constellations.
\end{abstract}

\section{Introduction}

In a communication system, blind equalization algorithm is introduced to the receiver in order to effectively eliminate the inter-symbol interference(ISI) caused by limited bandwidth and multi-path propagation. In blind equalization algorithms, constant modulus blind equalization algorithm(CMA) can make the output constellation points distribute in the circle with radius $R_{C}$ (statistical mode value of input signal) as far as possible, its cost function is only related to the received sequence amplitude, so CMA is very suitable to equalize constant modulus signals[1]. However, the different modulus constellation points of high order QAM or APSK signals are distributed in different radius of the circle. If CMA is used to equalize high order QAM or APSK signals, the output constellation points of high order QAM or APSK signals will be focused single circle, there will be large mean square error(MSE), and CMA even will become invalid. In recent years, some multi-modulus blind equalization algorithms(MMAs) were proposed to equalize multi-modulus signals. The MMAs can make the output constellation points of high order QAM or APSK signals equalize to different circles with different radii[2][3]. Compared with CMA, especially for non-square constellation and intensive constellation, MMA can make full use of the statistical characteristics of symbols. However, the MMA and CMA all exist the model error so that the convergence speed and residual MSE aren't still very ideal.

Memetic Algorithm is an optimization algorithm with genetic mechanism and local searching, can make all individuals obtain local optimal value after iteration, and is easy to understand and implement, as well as has simple operation [4][5].

According to characteristics of the MA and the MMA, a multi-modulus blind equalization algorithm based on MA is proposed. It can search global optimal group fast by MA, which is used 
as the initial optimal weight vector of the MA. The simulation results show that, the proposed algorithm has the ability to equalize higher-order multi-modulus signals, its convergence speed is faster and its steady MSE is smaller.

\section{Multi-modulus Blind Equalization Algorithm}

The MMA is shown in Figure 1. In Fig.1, a decision device is used to determine the decision modulus value $R_{D}$ of multi-modulus signals. $\boldsymbol{x}(n)$ is the receiving sequence to equalizer, $\boldsymbol{w}_{D}(n)$ is the weight vector of the equalizer, $z(n)$ is the output of the equalizer, $\hat{z}(n)$ is estimated signal of $z(n), e_{D}(n)$ is error signal, $R_{J}$ is sampling modulus value. After $z(n)$ passes nonlinear estimator without memory, the estimated sequence $\hat{z}(n)$ instead of the expected signal can be obtained. The memory less nonlinear function of the MMA is defined as

$\hat{z}(n)=g[z(n)]=z(n)\left[1+R_{D}^{2}-|z(n)|^{2}\right]$

$R_{J}$ is defined as

$R_{J}=\frac{E\left[|z(n)|^{4}\right]}{E\left[|z(n)|^{2}\right]}$

The output of the MMA is given by

$z(n)=\boldsymbol{w}^{\mathrm{T}}(n) \boldsymbol{x}(n)$

The error signal is defined as

$e_{D}(n)=\hat{z}(n)-z(n)=z(n)\left[R_{D}^{2}-|z(n)|^{2}\right]$

The cost function of the MMA is written as

$$
J_{M M A}(n)=E\left\{\left[R_{D}^{2}-|z(n)|^{2}\right]^{2}\right\}
$$

The weight vector iterative formula of the MMA is obtained by stochastic gradient and written as $\boldsymbol{w}_{D}(n+1)=\boldsymbol{w}_{D}(n)-\mu_{D} e_{D}(n) \boldsymbol{x}^{*}(n)$

where $\mu_{D}$ is step size factor.

According to decision modulus value $R_{D}$, MMA can adjust error function. For 32-APSK signal, its constellations can be known as the star QAM[6][7] and shown in Figure 2. In Figure2, the input constellation points are distributed in three different circles with different radii, two circles with the dashed border are decision boundary condition for decision device. The constellations are divided into three regions correspond to three error function by two decision circles.

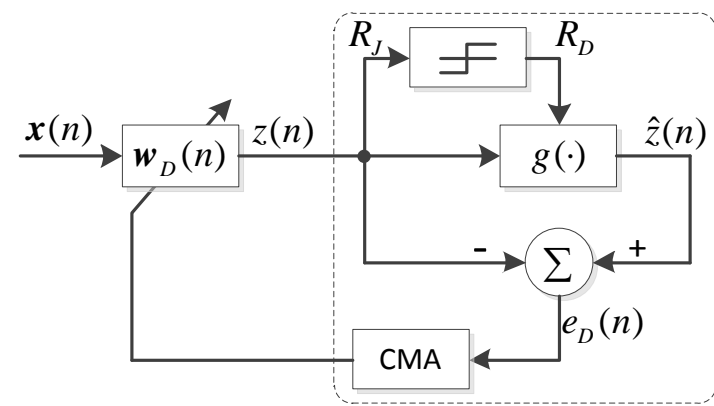

Fig. 1 MMA

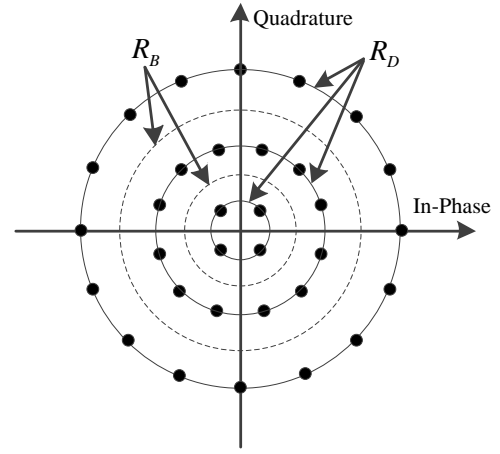

Fig. 2 32-APSK

In the constellation, the radius $R_{D}$ of circle are ranked as $R_{D 1}, R_{D 2}$, and $R_{D 3}$ in ascending order. The radius of decision circle in ascending order are $R_{s}, R_{b}$.

The equalizer output $z(n)$ is input to decision device. In order to obtain $R_{D}$ and $e_{D}(k)$, it is necessary to compare $R_{J}$ with the decision boundary $R_{s}$ and $R_{b}$. So we have 


$$
R_{D}=\left\{\begin{array}{l}
R_{D 1}, R_{J}<R_{s} \\
R_{D 2}, R_{s}<R_{J}<R_{b} \\
R_{D 3}, R_{J}>R_{b}
\end{array}\right.
$$

The error function of 32-APSK is given as

$$
e_{D}(n)=\left\{\begin{array}{l}
z(n)\left[R_{D 1}^{2}-|z(n)|^{2}\right], R_{J}<R_{s} \\
z(n)\left[R_{D 2}^{2}-|z(n)|^{2}\right], R_{s}<R_{J}<R_{b} \\
z(n)\left[R_{D 3}^{2}-|z(n)|^{2}\right], R_{J}>R_{b}
\end{array}\right.
$$

For different modulation modulus, the decision rules aren't the same. The equalization performance of the MMA mainly depends on the selection of decision condition $R_{s}$ and $R_{b}$. MMA has some advantages such as low computational complexity and the fast convergence speed, but its residual MSE is large and its convergence speed is slow.

\section{Correlation Algorithms}

Genetic algorithm. Genetic algorithm is a non deterministic quasi natural algorithm, which is a simulation of biological genetic and evolutionary process[8]. It starts from a population representative of a potential solution of possible problem, a new generation of population can be generated by genetic operator of nature genetics such as code, population initialization, and fitness calculation, as well as the hybrid and mutation. The whole process simulates the process of biological evolution in natural, the environmental adaptability of the newborn generation is stronger than that of previous generation. The decoded best individual in the last generation can be served as the approximate optimal solution of the problem.

Memetic algorithm. The word "Memetic" is derived from 'meme', which is generally understood as 'cultural gene'. Therefore, Memetic algorithm is called as a culture genetic algorithm, which simulates the development of human civilization. Its operation process is similar to the genetic algorithm, but its local search can make all individuals in each time of iteration reach the local optimal. The MA usually can be defined as the combined algorithm of evolution and personal learning in a much more diverse background [9]. The following piece of pseudo-code can make things more explicit.

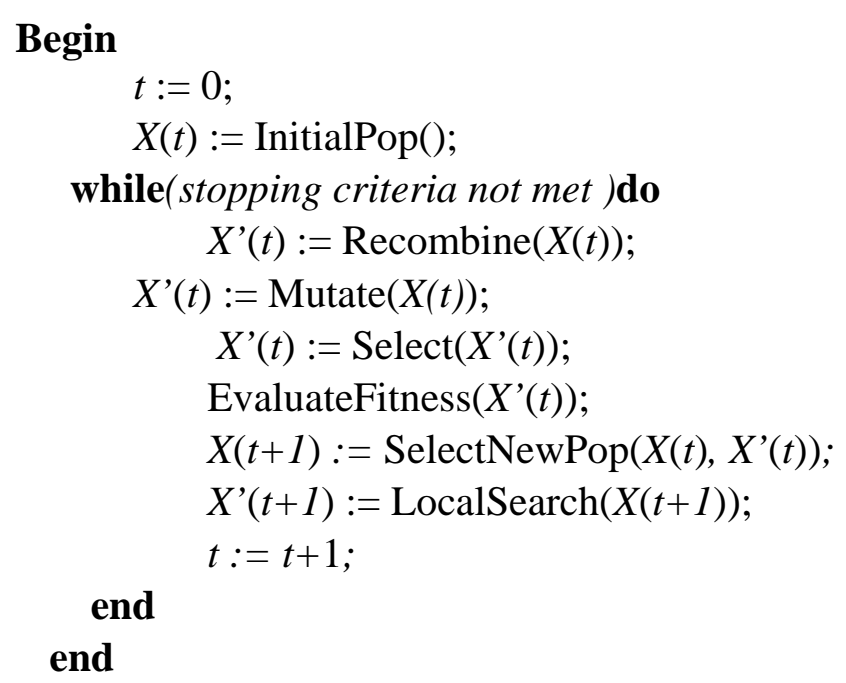

Step1: Code. Binary code is used.

Step2: Initialization of the population. The initial population is generated randomly (where $N$ is the population size) and denoted as $\boldsymbol{X}=\left[\boldsymbol{X}_{1}, \boldsymbol{X}_{2}, \cdots, \boldsymbol{X}_{N}\right]$.

Step3: Recombination. In accordance with the recombination probability $\left(P_{c}\right)$, two individuals have been selected randomly to generate two new individuals of new generation of population. 


$$
\begin{aligned}
& \boldsymbol{X}_{1}^{\prime}=\omega_{1} \boldsymbol{X}_{1}+\left(1-\omega_{1}\right) \boldsymbol{X}_{2} \\
& \boldsymbol{X}_{2}^{\prime}=\omega_{2} \boldsymbol{X}_{2}+\left(1-\omega_{2}\right) \boldsymbol{X}_{1}
\end{aligned}
$$

(10)

where $\boldsymbol{X}_{1}$ and $\boldsymbol{X}_{2}$ are randomly selected as two parent individuals, $\boldsymbol{X}_{1}^{\prime}$ and $\boldsymbol{X}_{2}^{\prime}$ are the corresponding new offspring individuals after recombination calculation, $\omega_{1}$ and $\omega_{2}$ are two random number in $[0,1]$.

Step 4: Mutation operator. After calculating according to certain rules, some individuals with mutation probability $\left(P_{m}\right)$ in new generation of population are selected. The mutate operation is given by

$$
\boldsymbol{X}^{\prime}=\left\{\begin{array}{l}
\boldsymbol{X}+\left(\boldsymbol{X}_{\text {max }}-\boldsymbol{X}\right)\left(\text { rand } \times g_{t}\right)^{2}, \text { sign }=0 \\
\boldsymbol{X}-\left(\boldsymbol{X}-\boldsymbol{X}_{\text {min }}\right)\left(\text { rand } \times g_{t}\right)^{2}, \text { sign }=1
\end{array}\right.
$$

where $g_{t}=t / G$ is the identification of the population evolution (where $t$ is the current evolution times, $G$ is the maximum of evolution times). $\boldsymbol{X}$ is the selected mutation individuals, $\boldsymbol{X}^{\prime}$ is mutated individuals, rand is a random number in [0,1], sign is set 0 or $1, \boldsymbol{X}_{\max }$ and $\boldsymbol{X}_{\min }$ are the upper and lower limits of the parameters, respectively.

Step 5: Evaluate fitness. Evaluate fitness is denoted as fitness $(\boldsymbol{X})$.

Step 6: Select operator. $N$ excellent individuals (high fitness value) in the current population are selected to make them enter next iteration and some individuals with low fitness value will be eliminated. Each probability $\left(P_{i}\right)$ of individual is defined as

$$
P_{i}=\frac{\text { fitness }\left(\boldsymbol{X}_{i}\right)}{\sum \text { fitness }\left(\boldsymbol{X}_{i}\right)} \quad, \quad i=1,2, \cdots N
$$

where fitness $\left(\boldsymbol{X}_{i}\right)$ is the ith individual fitness value.

Step 7: local search. The local search of all individuals is carried out by using the MATLAB built-in optimization function.

Step 8: Iteration end. When the current iteration number is greater than the maximum iteration time, the optimal solution is output, otherwise go to step 3.

Based on the mechanism of metaphor in the theory of cultural development, the MA is a collection of global search and heuristics local search, it is different from other intelligent optimization algorithms with specific mathematical model, and it is more of a thought or frame.

\section{Multi-Modulus Blind Equalization Algorithm Based on MA}

When the MA is introduced to the MMA, multi-modulus blind equalization algorithm based on MA(MA-MMA) is proposed and shown in Fig.3. In Fig.3, $s(n)$ is the transmitted signal, $n_{0}(n)$ is Gaussian white noise.

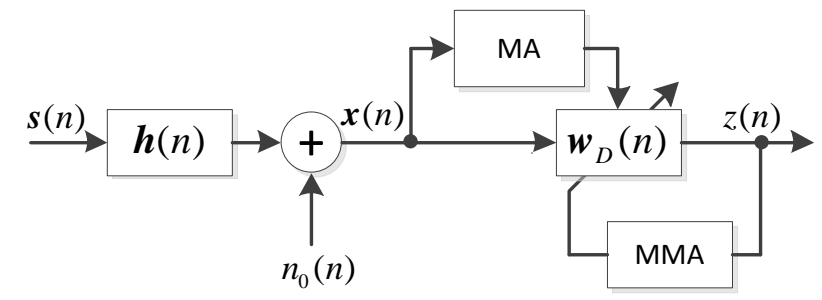

Fig.3 MA-MMA

Assume that the initial population of randomly generation is $\boldsymbol{X}=\left[\boldsymbol{X}_{1}, \boldsymbol{X}_{2}, \cdots, \boldsymbol{X}_{N}\right]$, each individual $\boldsymbol{X}_{i}$ corresponds to one of the weight vector of the MMA. The optimal weight vector of the MMA can be obtained by local search and global evolution. In the MA-MMA, the reciprocal of 
cost function of MMA is defined as the fitness function of the MA, i.e.

$$
\text { fitness }\left(\boldsymbol{X}_{i}\right)=\frac{1}{J_{M M A}\left(\boldsymbol{X}_{i}\right)} \quad i=1,2, \cdots N
$$

\section{Simulation and Analyses}

In order to verify the performance of the MA-MMA, the simulation test of the MA-MMA was carried out and compared with the CMA, MMA, and GA-MMA. In the test, $N=50, S=20$,

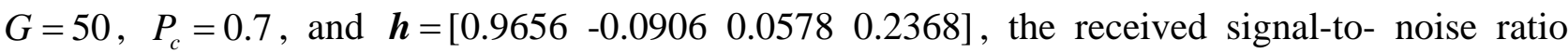
$S N R=30 \mathrm{~dB}$, the equalizer had 11 order transversal tap structure, the center tap coefficient of the CMA and MMA were set to 1, the other tap coefficient were set to 0, maximum iterations of all simulation iter $=10000$, Monte Carlo times $M=2000$. The step-sizes of the CMA and MMA were $1 \times 10^{-5}$, the step-sizes of the MA-MMA and GA-MMA were $5 \times 10^{-6}$, the simulation results were shown in Figure 4.

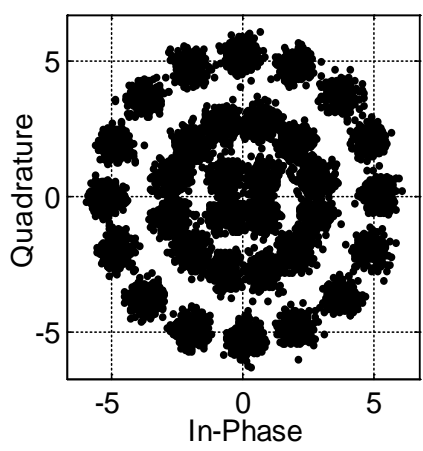

(a) Output of CMA

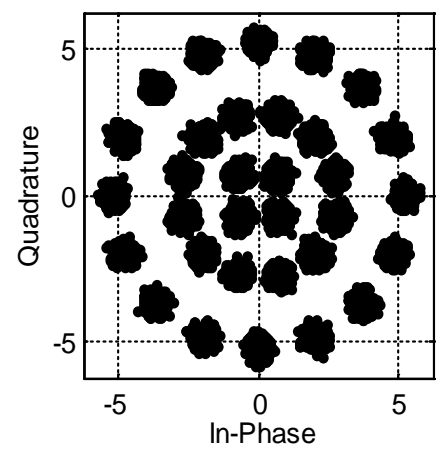

(b) Output of MMA

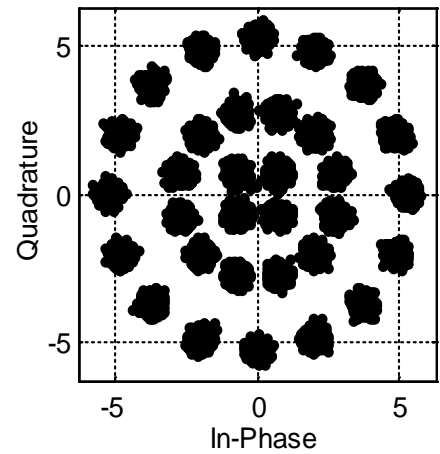

(c) Output of GA-MMA

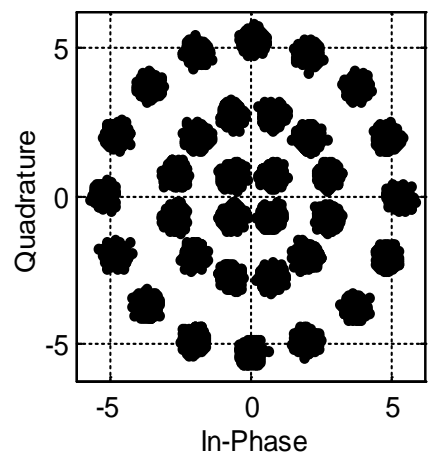

(d) Output of MA-MMA

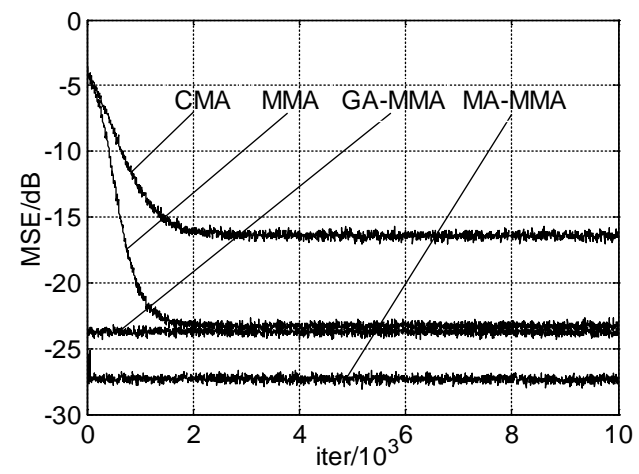

(e) The convergence curves

Fig. 4 Simulation results of 32-APSK

In the same SNR, the iterations and the steady MSE were shown in Table 1.

Tab. 1 Convergence iterations and steady MSE

\begin{tabular}{|c|c|c|}
\hline Algorithms & MSE/dB & Iterations \\
\hline CMA & -17 & 2200 \\
\hline MMA & -23 & 1800 \\
\hline GA-MMA & -24 & 100 \\
\hline MA-MMA & -27 & 100 \\
\hline
\end{tabular}

From Figure 4 and Table 1, we can come some following conclusions: (1) the equalization performance of the MA-MMA and the GA-MMA are much better than that of the MMA, the convergence speed of the MA-MMA is slightly faster than that of the GA-MMA, but its MSE is smallest. (2) the output constellations of the MA-MMA is more clearer and more compact than those of the CMA and MMA. Therefore, for higher-order multi-modulus signals, the MA-MMA 
outperforms the CMA, MMA, and GA-MMA in improving convergence speed and reducing steady MSE.

\section{Conclusions}

This paper proposes a multi-modulus blind equalization algorithm based on Memetic Algorithm. This proposed algorithm makes full use of the genetic mechanism and local searching of the MA to obtain the initial optimal weight vector, so that the equalization performance of the MMA can be improved. The simulation results show that, for higher-order multi-modulus 32-APSK signals, MA-MMA has faster convergence speed and smaller steady MSE comparison with traditional CMA, MMA, and GA-MMA.

\section{Acknowledgements}

Project supported the Major Project of Nature Science Foundation of Higher Education Institution of Jiangsu Province, China(Grant No.13KJA510001), Jiangsu Scientific Research Achievements in Industrialization Project, China(JHB 2012-9), and Jiangsu Province, the 2015 annual general university graduate students practice innovation program(SJLX15_0398)

\section{Reference}

[1] Gao Yuan, Qiu Xinyun. A New Variable Step Size CMA Blind Equalization Algorithm [C]//IEEE Chinese Control and Decision Conference, Taiyuan, China, 2012, 5: 315 - 317.

[2] Yang Jian, Jean-Jacques Werner, Guy A. Dumont. The Multi-modulus Blind Equalization and Its Generalized Algorithm[J]. IEEE Journal on Selected Areas in Communications,2002, 20(5): 9971014.

[3] Jenq-Tay Yuan. Equalization and Carrier Phase Recovery of CMA and MMA in Blind Adaptive Receivers[J]. IEEE Tran. on Signal Processing, 2010, 58(6): 3206 -3217.

[4] Swapna Devi, Devidas G. Jadhav, Shyam S. Pattnaik. Memetic Algorithm and Its Application to Function Optimization and Noise removal [C]//IEEE World Congress on Information and Communication Technologies, 2011, 749-753.

[5] Lin Geng, , Zhu Wenxing. An efficient Memetic Algorithm for the Max-Bisection Problem[J]. IEEE Trans. on Computers, 2014, 63(6): 1365-1376.

[6] Yao Ma, Zhang Q.T. Diversity Reception of DAPSK Over Generalized Fading Channels[J]. IEEE Trans. on Wireless Communications, 2005, 4(4): 1834 - 1845.

[7] Christian Hager, Alex Alvarado. Design of APSK Constellations for Coherent Optical Channels with Nonlinear Phase Noise[J]. IEEE Trans. on Communication, 2013, 61(8): 3362 - 3373.

[8] Kalyanmoy Deb, Amrit Pratap, Sameer Agarwal et al. A Fast and Elitist Multi-objective Genetic Algorithm: NSGA-II[J]. IEEE Trans. on Evolutionary Computation, 2002, 6(2): 182-197.

[9] Q. H. Nguyen, Y. S. Ong, N. Krasnogor. A Study on the Design Issues of Memetic Algorithm[C]//IEEE Congress on Evolutionary Computation, 2007, 2390-2397. 